\title{
Synthesis of new analogs of 3-methyl-[1,2,4] triazolo [3,4-a] phthalazines via Suzuki coupling and evaluation of their anticancer and antimicrobial activity
}

\author{
Gollapudi Ravi Kumar ${ }^{1,2}$, Chandra Mohan Kurmarayuni ${ }^{1}$, Manideepa Indupalli ${ }^{3}$, Ramya Krishna \\ Pallapati ${ }^{1}$ and Hari Babu Bollikolla ${ }^{1, *}$ \\ ${ }^{1}$ Department of Chemistry, Acharya Nagarjuna University, Nagarjuna Nagar-522510, Guntur, \\ Andhra Pradesh, India \\ ${ }^{2}$ Department of Chemistry, Government Degree College, Husnabad-505467, Telangana, India \\ ${ }^{3}$ Department of Botany \& Microbiology, Acharya Nagarjuna University, Nagarjuna Nagar-522510, Guntur, \\ Andhra Pradesh, India
}

\begin{abstract}
A series of new N-aryl substituted phenyl acetamide analogs of 3-methyl-[1,2,4] triazolo[3,4- $a$ ] phthalazines were synthesized starting from commercially available, in-expensive phthalic anhydride in good yields $(65-75 \%)$ via Suzuki Coupling. These compounds were tested for inhibition activity against HCT 116 cancer cell line by using MIT assay. Among the library of compounds, N-(3-methoxyphenyl)-2-(4-(3-methyl$[1,2,4]$ triazolo[3,4-a]phthalazin-6-yl)phenyl) acetamide followed by 2-(4-(3-methyl-[1,2,4]triazolo[3,4a]phthalazin-6-yl)phenyl)-N-(m-tolyl) acetamide and $\mathrm{N}$-(3-chlorophenyl)-2-(4-(3-methyl-[1,2,4]triazolo[3,4a]phthalazin-6-yl)phenyl) acetamide were found to be active compounds with $\mathrm{IC}_{50}$ of 70 to and $90 \mu \mathrm{g} \mathrm{mL}$. Further, the compounds were also screened for their antimicrobial activities.
\end{abstract}

Keywords: Phthalazine; Triazole; Anticancer; HCT 116; Antimicrobial activity.

\section{Introduction}

One among the clinical stage dependant deadliest disease is cancer. Cancer occupies its place next to cardiovascular disease and diabetes in decreasing the lifetime of a human being ${ }^{1}$. Recent statistics on cancer studies reveal the fact that several incidences of cancer and cancer deaths are crossing alarming levels. In 2012 globally 14.1 million new cancer cases were reported and out of which 8.2 million deaths were recorded ${ }^{2}$.

Heterocycles are relevant pharmacophoric units in the natural products as well as in the synthetic drug molecules. They found in various biologically active agents and exhibits diverse bio-activities. Among these compounds, nitrogen-containing heterocyclic compounds have good applications in the biological system such as pharmaceuticals, pyrotechnics, explosives and chemotherapy ${ }^{3}$. Among the nitrogencontaining heterocyclic compounds, phthalazine derivatives form a structural profile for biologically active molecules ${ }^{4-9}$, and hence they play a vital role in the biological systems. Phthalazine derivatives show significant biological activity such as antifungal ${ }^{10,11}$, antimicrobial and antitumor agents ${ }^{12,13}$. Azelastine (A, Fig. 1) MY5445 (B, Fig. 1) which are the phthalazine derivatives having the remarkable active drugs. Azelastine is used for the treatment of allergic rhinitis ${ }^{14}$; it is an antihistamine drug. MY5445 is a suitable inhibitor of cGMP-inhibited phosphodiesterase (PDE) leads to inhibition of human platelet aggregation ${ }^{15-18}$. The phthalazine derivative Zopolrestat (C, Fig. 1) has been in the clinical trials; in the prevention of neuropathy, retinopathy and cataract formation in diabetes ${ }^{19}$. The compound luminol (D, Fig. 1) has found in analytical applications like chemiluminescent reactions ${ }^{20-23}$.

Similarly, 1,2,4-triazole derivatives itself shows significant biological activities such as antiinflammatory ${ }^{24}$, anti-HIV ${ }^{25}$, antibacterial ${ }^{26,27}$ and antiplatelet ${ }^{28}$ activities. Recently these derivatives show the thymidine phosphorylase inhibiting activity ${ }^{29}$ Thymidine phosphorylase (TP) is over expressed in various solid tumors, and this inhibition activity can offer distinctive target suitable for the discovery of cancer drugs, and these derivatives have carbonic anhydrase II inhibition activity ${ }^{30}$. 
<smiles>CN1CCCC(Cn2nc(Cc3ccc(Cl)cc3)c3ccccc3c2=O)CC1</smiles>

A<smiles>O=C(O)Cc1nn(Cc2nc3cc(C(F)(F)F)ccc3s2)c(=O)c2ccccc12</smiles>

C<smiles>Clc1cccc(Nc2nnc(-c3ccccc3)c3ccccc23)c1</smiles>

B<smiles>Nc1cccc2c(=O)[nH]nc(O)c12</smiles>

D

Figure 1. Important Phthalazine derivatives

So, in the present study, we considered it is worth to design a new derivative series, which are the combination of phthalazine and 1,2,4-Triazole units for potential anticancer compounds.

Day by day, the numbers of synthetic drugs are found to be increasing and are validated against clinical stage dependent carcinomas. One of the widely studied colorectal cancer cell line in vitro is
HCT116. The tumor progression and drug resistance pathways are well reported in HCT116 CRC cell line 31,32 . In the present study as a continuous effort for developing new anticancer compounds 33-36 we synthesized and screened some phthalazine analogs with the above discussed structural units and reported their anticancer activity on HCT116 colon carcinoma cell line and antimicrobial activities on five different organisms.<smiles>O=C1OC(=O)c2ccccc21</smiles>

1<smiles>O=c1[nH][nH]c(=O)c2ccccc12</smiles>

2

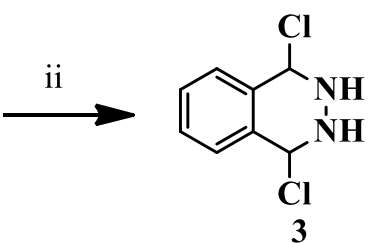
3<smiles>COC(=O)Cc1ccc(-c2nn3c(C)nnc3c3ccccc23)cc1</smiles><smiles>CCC</smiles><smiles>Cc1nnc2c3ccccc3c(-c3ccc(CC(=O)O)cc3)nn12</smiles><smiles>[R][R]C(=O)Cc1ccc(-c2nn3c(C)nnc3c3ccccc23)cc1</smiles>

$\mathrm{R}=8 \mathrm{a}: \mathrm{o}$ - methyl phenyl $8 \mathrm{~b}:$ m-methyl phenyl $8 \mathrm{c}:$ p- methyl phenyl 8d: o-methoxy phenyl 8e: m-methoxy phenyl 8f: p-methoxy phenyl 8g: o-chloro phenyl 8h: m-chloro phenyl 8i: p-chloro phenyl

Scheme 1. Synthetic protocol of 3-Methyl-[1,2,4]triazolo $[3,4-a]$ phthalazine analogs 
Reagents and conditions: (i): Hydrazine hydrate, $\mathrm{CH}_{3} \mathrm{COOH}, 120^{\circ} \mathrm{C}, 4 \mathrm{~h}, 83.77 \%$; (ii): $\mathrm{POCl}_{3}, 110^{\circ} \mathrm{C}$, 1h, $85.7 \%$; (iii): Hydrazine hydrate, $\mathrm{C}_{2} \mathrm{H}_{5} \mathrm{OH}, 78^{\circ} \mathrm{C}$, 1h, $81.84 \%$; (iv): $\mathrm{CH}_{3} \mathrm{COCl}$, Dioxane, $\mathrm{N}\left(\mathrm{C}_{2} \mathrm{H}_{5}\right)_{3}, 110$ ${ }^{\circ} \mathrm{C}$, 2h, $70.0 \%$; (v): (4-(2-methoxy-2-oxoethyl) phenyl) boronic acid, X-phos, $\mathrm{Pd}_{2}(\mathrm{dba})_{2}, \mathrm{~K}_{2} \mathrm{CO}_{3}$, $\mathrm{H}_{2} \mathrm{O}$, Dioxane, $110{ }^{\circ} \mathrm{C}, 3 \mathrm{~h}, 45.4 \%$; (vi): $\mathrm{KOH}$, $\mathrm{CH}_{3} \mathrm{OH}$, rt, 2h, $96.7 \%$; (vii) T3P, N,N-Diisopropylethylamine, DCM, rt, $3 \mathrm{~h}$.

\section{Results and Discussion}

The synthetic protocol for the synthesis of new analogs of 3-methyl-[1,2,4] triazolo[3,4-a] phthalazines has shown in Scheme 1. In the first step of the synthesis, commercially available, in-expensive phthalic anhydride (1) was reacted with hydrazine hydrate in acetic acid under reflux conditions afforded phthalazine (2) in excellent yield. In the next step, phthalazine was treated with phosphorus oxychloride at $110{ }^{\circ} \mathrm{C}$ to get 1,4 -dichlorophthalazine ${ }^{37-39}(3)$ in excellent yield. In step three of the synthetic sequence, compound 3 was treated with hydrazine hydrate in ethanol solvent at refluxing temperature produced 1-choro-4-hydrazinophthalazine (4) in very excellent yield. The obtained compound $\mathbf{4}$ was dissolved in dioxane, acetyl chloride and triethylamine were added. The reaction mixture was subjected to reflux for $12 \mathrm{~h}$, to get the compound $\mathbf{5}$ in good yield.

Upon achieving the compound $\mathbf{5}$, it was treated with Boronic acid Tris(dibenzylidene acetone) dipalladium (0) $\left(\mathrm{Pd}_{2}(\mathrm{dba})_{2}\right)$, compound $\mathbf{6}$ was obtained in poor yield through Suzuki reaction. The formed ester derivative (6) undergoes ester hydrolysis by using potassium hydroxide in methanol as solvent yields acid derivative (7) in quantitative yield. Finally, the novel acid derivative on reaction with various aromatic amines and propylphosphonic anhydride $\left(\mathrm{T}_{3} \mathrm{P}\right)$ reagent in DCM solvent yields compounds 8a$\mathbf{8 i}$, and the results were mentioned in Table 1 .

Table 1. Synthetic and In vitro anticancer activity results of the compounds 7, 8a-8i.

\begin{tabular}{|l|l|l|l|}
\hline Compound & R- & Yield \% & IC50 in $\mathbf{~} \mathbf{g} \mathbf{~ m L}^{-\mathbf{1}}$ \\
\hline $\mathbf{8 a}$ & $o$-methyl phenyl & 65.2 & 162.4 \\
\hline $\mathbf{8 b}$ & m-methyl phenyl & 69.2 & 74.6 \\
\hline $\mathbf{8 c}$ & $p$-methyl phenyl & 72.2 & 98.6 \\
\hline $\mathbf{8 d}$ & $o$-methoxy phenyl & 74.1 & 125.1 \\
\hline $\mathbf{8 e}$ & m-methoxy phenyl & 70.1 & 57.4 \\
\hline $\mathbf{8 f}$ & $p$-methoxy phenyl & 66.2 & 101.1 \\
\hline $\mathbf{8 g}$ & $o$-chloro phenyl & 78.2 & 132.9 \\
\hline $\mathbf{8 h}$ & m-chloro phenyl & 68.12 & 75.6 \\
\hline $\mathbf{8 i}$ & $p$-chloro phenyl & 75.22 & 98.6 \\
\hline $\mathbf{7}$ & - & 96.7 & $>200$ \\
\hline Doxorubicin & - & - & 0.260 \\
\hline
\end{tabular}

The compound $\mathbf{8 g}$ with the ortho-chloro substitution was obtained in a higher yield of $78.2 \%$ and followed by compounds $\mathbf{8 i}$ and $\mathbf{8 d}$ of about $75 \%$ and $74 \%$ yield with para- chloro and ortho- methoxy substitutions respectively. The compound 8a with ortho- methyl substitution was found to be obtained with the lowest yield.

All the molecules were tested for their anticancer activity on HCT116 cancer cell line, and results were presented in Table 1.

The compounds with a meta-methoxy substitution (8e) were found to possess the highest activity $\left(\mathrm{IC}_{50}=57.4 \mu \mathrm{g} \mathrm{mL}^{-1}\right.$ ) moreover it was also observed that the compounds $\mathbf{8 b}$ and $\mathbf{8 h}$ with metamethyl and meta- chloro substitutions were found to possess almost equal activities $\left(\mathrm{IC}_{50}=74.6 ; 75.6 \mu \mathrm{g}\right.$ $\mathrm{mL}^{-1}$ ). Similarly, the compound $\mathbf{8 c}$ and $\mathbf{8 i}$ with para methyl and para- chloro substitutions were also shown similar activity, i.e. $\mathrm{IC}_{50}=98.6 \mu \mathrm{g} \mathrm{mL}-1$. Further, close observation on the substituted compounds showed the highest activity for metasubstitutions followed by para- substituted compounds. The ortho- substituted compounds possess the lowest activity. Further, compound 7 with acid functionality before conversion into its amide possesses activity $>200 \mu \mathrm{g} \mathrm{mL} \mathrm{mL}^{-1}$, which established the importance of the present structural unit.

The compounds were screened for their antimicrobial activities on five different pathogens, and the results were presented in Table 2. The results showed that the acid compound $\mathbf{7}$ displayed a significant activity on Candida albicans (with a zone of inhibition of $0.8 \mathrm{Cm}$ ) followed by compounds $\mathbf{8 h}$ and $8 \mathbf{a}$ with a zone of inhibition zones 0.7 and $0.6 \mathrm{Cm}$ respectively, whereas the positive tetracycline control was showed a zone of inhibition of $1.3 \mathrm{Cm}$. The compounds $8 \mathbf{c}$ and $\mathbf{8 h}$ were found to be moderately active against Staphylococcus aureus when compared to Tetracycline. Similarly, the compounds $\mathbf{7}$ and $\mathbf{8 f}$ were also moderately active against Escherichia coli. 
Table 2. The results of antimicrobial activities of the phthalazine derivatives.

\begin{tabular}{|l|l|l|l|l|l|}
\hline \multirow{2}{*}{ Comp. } & \multicolumn{2}{l}{ Zone of inhibition $(\mathbf{c m})$} \\
\cline { 2 - 6 } & SA & $\mathbf{B M}$ & PA & CA & EC \\
\hline $\mathbf{8 a}$ & 0.5 & 0.5 & 0.5 & 0.6 & - \\
\hline $\mathbf{8 b}$ & 0.5 & 0.6 & 0.6 & 0.4 & - \\
\hline $\mathbf{8 c}$ & 0.9 & 0.5 & 0.5 & 0.2 & - \\
\hline $\mathbf{8 d}$ & 0.6 & 0.6 & 0.6 & 0.2 & 0.5 \\
\hline $\mathbf{8 e}$ & - & - & - & - & - \\
\hline $\mathbf{8 f}$ & 0.5 & 0.5 & - & 0.2 & 0.7 \\
\hline $\mathbf{8 g}$ & 0.5 & 0.5 & - & - & 0.5 \\
\hline $\mathbf{8 h}$ & 0.8 & 0.8 & 0.5 & 0.7 & 0.6 \\
\hline $\mathbf{8 i}$ & 0.5 & 0.5 & - & 0.5 & - \\
\hline $\mathbf{7}$ & 0.5 & 0.5 & 0.8 & 0.8 & 0.8 \\
\hline Tetracycline & 1.6 & 1.7 & 1.7 & 1.3 & 1.6 \\
\hline
\end{tabular}

SA - Staphylococcus aureus; BA - Bacillus Megaterium; PA-Pseudomonas aeuroginosa;

CA-Candida albicaus; EC - Escherichia coli.

\section{Conclusion}

The synthesized novel target molecules were tested against the Human carcinoma cell line. Among the synthesized compounds N-(3-methoxyphenyl)-2(4-(3-methyl-[1,2,4]triazolo[3,4-a]phthalazin-6yl)phenyl) acetamide showed prominent activity, 2-(4-(3-methyl-[1,2,4]triazolo[3,4-a]phthalazin-6yl)phenyl)-N-(o-tolyl) acetamide and $\mathrm{N}$-(3-chlorophenyl)-2-(4-(3-methyl-[1,2,4]triazolo[3,4-

a]phthalazin-6-yl)phenyl) acetamide compounds showed good activity at nearly same concentration. Among the novel derivatives, meta-substituted derivatives showed better results than the other compounds. After to meta-substituted compounds para substituted derivatives showed average results against HCT 116 cell line. However, ortho substituted compounds showed poor results. The compounds 2-(4-(3-methyl-[1,2,4]triazolo[3,4-a]phthalazin-6yl)phenyl)acetic acid, N-(3-chlorophenyl)-2-(4-(3methyl-[1,2,4]triazolo[3,4-a]phthalazin-6-yl)phenyl) acetamide , 2-(4-(3-methyl-[1,2,4]triazolo[3,4a]phthalazin-6-yl)phenyl)-N-(p-tolyl) acetamide, and $\mathrm{N}$-(4-methoxyphenyl)-2-(4-(3-methyl-[1,2,4]-

triazolo[3,4-a]phthalazin-6-yl)phenyl) acetamide were significant in respect of antimicrobial activities.

\section{Experimental}

The progress of the reactions was monitored by thin layer silica gel plates (Merck Manufactures). All the compounds synthesized (8a-8i) were characterized by ${ }^{1} \mathrm{H}$ NMR (at $400 \mathrm{MHz}$ ), ${ }^{13} \mathrm{C}$ NMR (at $100 \mathrm{MHz}$ ) with Tetramethylsilane as an internal standard and HRMS spectral data.

\section{Preparation of 2,3-di hydro phthalazine-1,4-dione} (2)

The phthalic anhydride $(6.01 \mathrm{~g} 40 \mathrm{mmol})$ was taken in $25 \mathrm{~mL}$ of the acetic acid solution, and the mixture was heated to $60{ }^{\circ} \mathbf{C}$. At this stage $(2.8 \mathrm{~mL}, 44 \mathrm{mmol})$ of hydrazine hydrate was added slowly and was heated to $120{ }^{\circ} \mathbf{C}$ for 4 hours. TLC observed the completion of the reaction, and the reaction mixture was cooled to room temperature after completion. The formed precipitate was filtered through Buckner funnel. Later the precipitate was washed with petroleum ether (2 x $320 \mathrm{~mL})$ and dried under vacuum, the compound $2(5.50 \mathrm{~g}, 83.77 \%)$ was obtained as white solid. M.p. $181-183{ }^{\circ} \mathrm{C}$.

${ }^{1} \mathrm{H}$ NMR (300 MHz, DMSO): $\delta$ 7.59-7.63 (m, 2H), $8.14(\mathrm{~d}, J=7.9 \mathrm{~Hz}, 2 \mathrm{H}), 9.04(\mathrm{~s}, 2 \mathrm{H})$.

\section{Preparation of 1,4-dichlorophthalazine (3)}

The compound (2) (2.0 g $12 \mathrm{mmol})$ was added to a stirred solution of Phosphoryl chloride $(5 \mathrm{~mL})$. Now the reaction mixture was heated to $110{ }^{\circ} \mathbf{C}$ until compound $\mathbf{2}$ was completely soluble. TLC monitored the reaction completion. The reaction mixture cooled to room temperature. The reaction mixture was poured in crushed ice which was taken in ethyl acetate $(500 \mathrm{~mL})$ with continuous stirring. Then the ethyl acetate layer was separated and neutralised with triethylamine and then washed with water $(2 \times 500$ $\mathrm{mL})$. The ethyl acetate layer was separated, dried with $\mathrm{Na}_{2} \mathrm{SO}_{4}$ and concentrated under vacuum, the compound 3 (2.1 g, $85.7 \%$ ) was obtained as white solid. Mp: $192-193{ }^{\circ} \mathrm{C}$;

${ }^{1} \mathrm{H}$ NMR (400 MHz, DMSO) $\delta 8.29(\mathrm{~d}, J=7.6 \mathrm{~Hz}$, $1 \mathrm{H}), 8.11-7.94$ (m, 3H).

\section{Preparation of 1-chloro-4-hydrazinyl Phthalazine (4)}

The compound (3) $(2.0 \mathrm{~g}, 10 \mathrm{mmol})$ was dissolved in ethanol $(10 \mathrm{~mL})$. Hydrazine hydrate $(3.73 \mathrm{~mL}$, $76.5 \mathrm{mmol}$ ) was added to the reaction mixture, heated to reflux for $0.54 \mathrm{~h}$. TLC monitored reaction completion. The reaction mixture was cooled to room temperature and filtered through Buckner funnel. The filter cake was washed with diethyl ether $(2 \times 10 \mathrm{~mL})$ 
and dried under vacuum, the compound 4 (1.6 g, $81.84 \%$;) was obtained. Mp: $259-260{ }^{\circ} \mathrm{C}$;

${ }^{1} \mathrm{H}$ NMR (400 MHz, DMSO) $\delta 8.32-8.27(\mathrm{~m}, 1 \mathrm{H})$, $8.21(\mathrm{~s}, 1 \mathrm{H}, \mathrm{NH}), 8.07-8.03(\mathrm{~m}, 1 \mathrm{H}), 8.03-7.99(\mathrm{~m}$, 1H), 7.98 - 7.95 (m, 1H), 7.89 (s, 2H, $\left.\mathrm{NH}_{2}\right)$.

\section{Preparation of 6-chloro-3-methyl-[1,2,4]-triazolo- [3,4-a]phthalazine (5)}

The compound (4) (1.6 g, $8.22 \mathrm{mmol})$ was dissolved in Dioxane. TEA $(1.14 \mathrm{~mL}, 8.22 \mathrm{mmol})$ and acetyl chloride $(9.868 \mathrm{mmol})$ were added. The reaction mixture was heated to $110{ }^{\circ} \mathrm{C}$. TLC monitored reaction completion. The solvent was evaporated, left solid mixtures portioned between DCM $(200 \mathrm{~mL})$ and water $(3 \times 100 \mathrm{~mL})$. The DCM layer was dried with $\mathrm{Na}_{2} \mathrm{SO}_{4}$, filtered and concentrates on yielding a solid. The obtained compound purified by silica gel column chromatography to give compound (5) $(1.25 \mathrm{~g}, 70 \%)$. Mp: $169-170{ }^{\circ} \mathrm{C}$;

${ }^{1} \mathrm{H}$ NMR $(400 \mathrm{MHz}, \mathrm{CDCl} 3) \delta 8.64(\mathrm{~d}, J=7.9 \mathrm{~Hz}$, $1 \mathrm{H}), 8.24(\mathrm{~d}, J=8.2 \mathrm{~Hz}, 1 \mathrm{H}), 7.99(\mathrm{t}, J=7.6 \mathrm{~Hz}, 1 \mathrm{H})$, $7.85(\mathrm{t}, J=7.8 \mathrm{~Hz}, 1 \mathrm{H}), 2.81\left(\mathrm{~s}, 3 \mathrm{H}, \mathrm{CH}_{3}\right)$.

Preparation of methyl 2-(4-(3-methyl-[1,2,4]triazolo[3,4-a]phthalazin-6-yl)phenyl)acetate (6)

The compound (5) (1.25 g, $5.71 \mathrm{mmol})$ was added to a stirred solution of dry Dioxane $(10 \mathrm{~mL})$. Later the Boronic acid $(1.781 \mathrm{~g} 8.565 \mathrm{mmol})$ was added to the stirred solution. Further, X-phos $(0.6 \mathrm{mmol})$ was added to the above mixture, potassium carbonate (11.42 mmol) and $1 \mathrm{~mL}$ water were added. Degassing the reaction mixture using Nitrogen gas. Tris(dibenzylideneacetone)dipalladium $(0)$

$\left(\operatorname{Pd}_{2}(\mathrm{dba})_{2}\right)(0.2 \mathrm{mmol})$ was added to the reaction mixture. Then the mixture was heated to reflux $110{ }^{\circ} \mathrm{C}$ for $3 \mathrm{~h}$. The TLC monitored the reaction completion. The reaction mixture filtered through celite bed. Later the solvent present in the filtrate was removed under reduced pressure, purification carried by silica gel column chromatography to yield compound 6 ( $0.9 \mathrm{~g}$, $45.4 \%)$.

Methyl-2-(4-(3-methyl-[1,2,4]triazolo[3,4-a]phthalazin-6-yl)phenyl)acetate (6)

Brown solid, yield 45.4\%; Mp: 151-153 ${ }^{\circ} \mathrm{C} ; \mathrm{R}_{\mathrm{f}} 0.6$ (methanol: dichloro methane, 0.05: 1.0);

${ }^{1} \mathrm{H}$ NMR (400 MHz, DMSO) $\delta 8.59(\mathrm{~d}, J=7.02 \mathrm{~Hz}$, $1 \mathrm{H}), 8.08-8.04(\mathrm{~m}, 1 \mathrm{H}), 7.87(\mathrm{~d}, J=4 \mathrm{~Hz}, 2 \mathrm{H}), 7.68$ $(\mathrm{d}, J=4 \mathrm{~Hz}, 2 \mathrm{H}), 7.53(\mathrm{~d}, J=4 \mathrm{~Hz}, 2 \mathrm{H}), 4.10(\mathrm{~s}, 3 \mathrm{H})$, 3.75 (s, 2H), 2.72 (s, 3H).

${ }^{13} \mathrm{C}$ NMR (100 MHz, DMSO) $\delta 171.9,154.3,152.8$, 146.1, 144.1, 135.7, 135.9, 132.8, 132.5, 127.2, 125.2, $125.0,122.9,121.9,120.7,120.3,118.2,53.9,44.2$, 9.9 .

HRMS (ESI): Calcd. [M+H] $]^{+} \mathrm{m} / \mathrm{z}$ : 333.1280, Found: 333.1273 .
Preparation of 2-(4-(3-methyl-[1,2,4]triazolo[3,4a]phthalazin-6-yl)phenyl)acetic acid (7)

The compound (6) $(0.9 \mathrm{~g}, 12.60 \mathrm{mmol})$ was dissolved in methyl alcohol, and $\mathrm{KOH}(2.60 \mathrm{mmol})$ was added to the reaction mixture. The reaction mixture was stirred at room temperature for $2 \mathrm{~h}$. The reaction completion was monitored by the TLC. The reaction mixture is neutralised with $2 \mathrm{M} \mathrm{HCl}$ formed solid was filtered. The obtained compound $7(0.8 \mathrm{~g}, 96.7 \%$;) is in brown.

\section{2-(4-(3-methyl-[1,2,4]triazolo[3,4-a]phthalazin-6- yl)phenyl)acetic acid (7)}

Brown solid, yield 96.7 \%; Mp: 211-213 ${ }^{\circ} \mathbf{C} ; \mathrm{R}_{\mathrm{f}} 0.30$ (methanol: dichloro methane, $0.1: 1.0$ );

${ }^{1} \mathrm{H}$ NMR (400 MHz, DMSO) $\delta 12.43(\mathrm{~s}, 1 \mathrm{H}), 8.56$ $(\mathrm{d}, \mathrm{J}=4 \mathrm{~Hz}, 1 \mathrm{H}), 8.06-8.00(\mathrm{~m}, 1 \mathrm{H}), 7.85-7.82$ $(\mathrm{m}, 2 \mathrm{H}), 7.65(\mathrm{~d}, J=7.6 \mathrm{~Hz}, 2 \mathrm{H}), 7.50(\mathrm{~d}, J=7.6 \mathrm{~Hz}$, 2H), 3.72 (s, 2H), 2.69 (s, 3H).

${ }^{13} \mathrm{C}$ NMR (100 MHz, DMSO) $\delta 177.8,155.5,153.8$, 146.9, 144.4, 135.9, 132.9, 132.5, 127.4, 125.2, 125.1, 123.0, 121.9, 120.7, 120.3, 118.3, 49.1, 9.6.

HR-MS(ESI): Calcd. [M+H] $]^{+} \mathrm{m} / \mathrm{z}: 319.1156$, Found: 319.1184 .

\section{General procedure for the synthesis of $8 \mathrm{a}$ to $8 \mathrm{i}$}

The compound 7 (50 mg $0.157 \mathrm{mmol}$ ) was added to a stirred solution of DCM (3 mL). After dissolving propyl phosphonic anhydride solution $\left(\mathrm{T}_{3} \mathrm{P}\right)$ $(0.157 \mathrm{mmol})$ was added to the reaction mixture. $\mathrm{N}$, $\mathrm{N}$ - Diisopropyl ethyl amine $(0.188 \mathrm{mmol})$ was added to the reaction mixture. The reaction mixture was stirred at room temperature for $3 \mathrm{~h}$. After the reaction completion, the reaction mixture was portioned between DCM $(1 \times 20 \mathrm{~mL})$ and water $(2 \times 10 \mathrm{~mL})$. The DCM layer was dried with $\mathrm{Na}_{2} \mathrm{SO}_{4}$, filtered and concentrated under reduced pressure. The residue obtained was purified by silica gel column chromatography to give $8 \mathrm{a}-8 \mathrm{i}$ with good yield, ${ }^{1} \mathrm{H}$ NMR, HR-MS(ESI) data of each compound were given below.

\section{2-(4-(3-methyl-[1,2,4]triazolo[3,4-a]phthalazin-6- $y l)$ phenyl)- $N$-(o-tolyl) acetamide (8a)}

Brown solid, yield 65.2\%; Mp: $163-165{ }^{\circ} \mathrm{C} ; \mathrm{R}_{\mathrm{f}} 0.55$ (methanol: dichloro methane, 0.1: 1.0);

${ }^{1} \mathrm{H}$ NMR (400 MHz, DMSO) $\delta 9.55(\mathrm{~s}, 1 \mathrm{H}), 8.57$ $(\mathrm{d}, J=8 \mathrm{~Hz}, 1 \mathrm{H}), 8.069-8.028(\mathrm{~m}, 1 \mathrm{H}), 7.87-7.84$ $(\mathrm{m}, 2 \mathrm{H}), 7.68(\mathrm{~d}, J=8 \mathrm{~Hz}, 2 \mathrm{H}), 7.60(\mathrm{~d}, J=8 \mathrm{~Hz}, 2 \mathrm{H})$, $7.40(\mathrm{~d}, J=8 \mathrm{~Hz}, 1 \mathrm{H}), 7.40(\mathrm{~d}, J=8 \mathrm{~Hz}, 1 \mathrm{H}), 7.20(\mathrm{~d}$, $J=8 \mathrm{~Hz}, 1 \mathrm{H}), 7.15(\mathrm{t}, J=8 \mathrm{~Hz}, 1 \mathrm{H}), 7.073(\mathrm{t}, J=8$ $\mathrm{Hz}, 1 \mathrm{H}), 3.82$ (s, 2H), 2.69 (s, 3H), 3.82 (s, 3H).

${ }^{13} \mathrm{C}$ NMR (100 MHz, DMSO) $\delta 169.1,156.5,147.8$, 142.2, 138.7, 136.6, 134.3, 132.2, 131.2, 130.7, 129.8, $129.1,126.3,125.7,123.8,122.9,122.8,42.9,18.3$, 9.9.

HR-MS(ESI): Calcd. $[\mathrm{M}+\mathrm{H}]^{+} \mathrm{m} / \mathrm{z}: 408.1355$, found: 408.1368 . 


\section{2-(4-(3-methyl-[1,2,4]triazolo[3,4-a]phthalazin-6- $y l)$ phenyl)-N-(m-tolyl) acetamide (8b)}

Brown solid, yield $69.2 \%$; Mp: $160-162{ }^{\circ} \mathrm{C}$; $\mathrm{R}_{\mathrm{f}} 0.55$ (methanol: dichloro methane, 0.1: 1.0);

${ }^{1} \mathrm{H}$ NMR (400 MHz, DMSO) $\delta 10.14(\mathrm{~s}, 1 \mathrm{H}, \mathrm{NH})$, $8.56(\mathrm{~d}, J=8 \mathrm{~Hz}, 1 \mathrm{H}, \mathrm{ArH}), 8.06-8.02(\mathrm{~m}, 1 \mathrm{H}, \mathrm{ArH})$, $7.83(\mathrm{~d}, J=4 \mathrm{~Hz}, 2 \mathrm{H}, \mathrm{ArH}), 7.67(\mathrm{~d}, J=8 \mathrm{~Hz}, 2 \mathrm{H}$, ArH), 7.57 (d, $J=8 \mathrm{~Hz}, 2 \mathrm{H}, \operatorname{ArH}), 7.45$ (s, 1H, ArH), $7.39(\mathrm{~d}, J=8 \mathrm{~Hz}, 1 \mathrm{H}, \operatorname{ArH}), 7.17(\mathrm{t}, J=8 \mathrm{~Hz}, 1 \mathrm{H}$, ArH), 6.85 (d, J=8 Hz, 1H, ArH), 3.77 (s, 2H, $\mathrm{CH}_{2}$ ), $2.68\left(\mathrm{~s}, 3 \mathrm{H}, \mathrm{CH}_{3}\right), 2.26\left(\mathrm{~s}, 3 \mathrm{H}, \mathrm{CH}_{3}\right)$,

${ }^{13} \mathrm{C}$ NMR (100 MHz, DMSO) $\delta 169.1,156.5,147.8$, $142.2,139.5,138.5,138.3,134.3,132.9,131.2,130.2$, $129.9,129.1,129.0,124.4,123.7,122.9,122.8,120.1$, 116.8, 43.6, 21.6, 9.9.

HR-MS(ESI): Calcd. $[\mathrm{M}+\mathrm{H}]^{+} \mathrm{m} / \mathrm{z}: 408.1355$, found: 408.1323

\section{2-(4-(3-methyl-[1,2,4]triazolo[3,4-a]phthalazin-6- yl)phenyl)-N-(p-tolyl) acetamide (8c)}

Light cream solid, yield $72.2 \%$; Mp: $166-168{ }^{\circ} \mathrm{C} ; \mathrm{R}_{\mathrm{f}}$ 0.55 (methanol: dichloro methane, 0.1: 1.0);

${ }^{1} \mathrm{H}$ NMR (400 MHz, DMSO) $\delta 10.13(\mathrm{~s}, 1 \mathrm{H}, \mathrm{NH})$, $8.56(\mathrm{~d}, J=8 \mathrm{~Hz}, 1 \mathrm{H}, \mathrm{ArH}), 8.06-8.02(\mathrm{~m}, 1 \mathrm{H}, \mathrm{ArH})$, $7.84(\mathrm{~d}, J=4 \mathrm{~Hz}, 2 \mathrm{H}, \operatorname{ArH}), 7.67(\mathrm{~d}, J=8 \mathrm{~Hz}, 2 \mathrm{H}$, ArH), 7.57 (d, $J=8 \mathrm{~Hz}, 2 \mathrm{H}, \operatorname{ArH}), 7.49(\mathrm{~d}, J=8 \mathrm{~Hz}$, $2 \mathrm{H}, \mathrm{ArH}), 7.09$ (d, $J=8 \mathrm{~Hz}, 2 \mathrm{H}, \mathrm{ArH}), 3.76(\mathrm{~s}, 2 \mathrm{H}$, $\left.\mathrm{CH}_{2}\right), 2.65$ (s, 3H, $\left.\mathrm{CH}_{3}\right), 2.23$ (s, 3H, $\mathrm{CH}_{3}$ ).

${ }^{13} \mathrm{C}$ NMR (100 MHz, DMSO) $\delta 169.2,156.5,147.9$, 142.3, 139.6, 138.6, 134.4, 133.0, 131.4, 131.0, 129.5, $129.2,129.0,124.6,123.9,123.0,122.9,120.0,116.9$, 43.9, 21.8, 9.9.

HR-MS(ESI): Calcd. $[\mathrm{M}+\mathrm{H}]^{+} \mathrm{m} / \mathrm{z}: 408.1355$, found: 408.1357.

\section{N-(2-methoxyphenyl)-2-(4-(3-methyl- [1,2,4]triazolo[3,4-a]phthalazin-6-yl)phenyl) acetamide $(8 d)$}

Dark brown solid, yield $74.1 \%$; Mp: $175-177{ }^{\circ} \mathbf{C}$; $\mathrm{R}_{\mathrm{f}}$ 0.40 (methanol: dichloro methane, 0.1: 1.0); ${ }^{1} \mathrm{H}$ NMR (400 MHz, DMSO) $\delta 9.39(\mathrm{~s}, 1 \mathrm{H}), 8.54(\mathrm{~d}, J=8 \mathrm{~Hz}$, $1 \mathrm{H}), 8.05-8.01(\mathrm{~m}, 1 \mathrm{H}), 7.94(\mathrm{~d}, J=4 \mathrm{~Hz}, 2 \mathrm{H}), 7.82$ (d, $J=4 \mathrm{~Hz}, 2 \mathrm{H}, \operatorname{ArH}), 7.67(\mathrm{~d}, J=8 \mathrm{~Hz}, 2 \mathrm{H}, \operatorname{ArH})$, 7.07 to $7.02(\mathrm{~m}, 2 \mathrm{H}, \mathrm{ArH}), 6.86$ to $6.90(\mathrm{~m}, 1 \mathrm{H}), 3.89$ $(\mathrm{s}, 2 \mathrm{H}), 3.83(\mathrm{~s}, 3 \mathrm{H}), 3.83(\mathrm{~s}, 3 \mathrm{H}), 2.68(\mathrm{~s}, 3 \mathrm{H}),{ }^{13} \mathrm{C}$ NMR (400 MHz, DMSO) $\delta 169.0,156.6,150.1$, 147.9, 142.3, 138.7, 134.3, 131.3, 130.2, 130.0, 129.2, 127.7, 125.0, 123.8, 123.0, 122.9, 122.3, 120.7, 111.6, 56.2, 43.2, 9.9. HR-MS(ESI): Calcd. $[\mathrm{M}+\mathrm{H}]^{+} \mathrm{m} / \mathrm{z}$ : 424.1280, found: 424.1225 .

\section{N-(3-methoxyphenyl)-2-(4-(3-methyl- [1,2,4]triazolo[3,4-a]phthalazin-6-yl)phenyl) acetamide $(8 \mathrm{e})$}

White solid, yield 70.1\%; Mp: 172-174 ${ }^{\circ} \mathbf{C}$; $\mathrm{R}_{\mathrm{f}} 0.40$ (methanol: dichloro methane, 0.1: 1.0); ${ }^{1} \mathrm{H}$ NMR (400 MHz, DMSO) $\delta 10.19(\mathrm{~s}, 1 \mathrm{H}), 8.56(\mathrm{~d}, J=8 \mathrm{~Hz}, 1 \mathrm{H})$, 8.06-8.02 (m, 1H), $7.84(\mathrm{~d}, J=3.6 \mathrm{~Hz}, 2 \mathrm{H}), 7.67(\mathrm{~d}, J$ $=8 \mathrm{~Hz}, 2 \mathrm{H}), 7.57(\mathrm{~d}, J=8.4 \mathrm{~Hz}, 2 \mathrm{H}), 7.32(\mathrm{~s}, 1 \mathrm{H})$, $7.22-7.13(\mathrm{~m}, 2 \mathrm{H}), 6.62(\mathrm{~d}, J=4 \mathrm{~Hz}, 1 \mathrm{H}), 3.78$ (s, 2H), $3.71(\mathrm{~s}, 3 \mathrm{H}), 2.65(\mathrm{~s}, 3 \mathrm{H}) .{ }^{13} \mathrm{C}$ NMR (400 MHz, DMSO) $\delta 169.3,160.0,156.5,147.9,142.3$, $140.8,138.4,134.4,133.0,131.3,130.2,130.0,129.9$, 129.2, 123.8, 122.9, 122.8, 122.7, 111.9, 109.3, 105.2, 55.4, 43.6, 9.9. HR-MS(ESI): Calcd. $[\mathrm{M}+\mathrm{H}]^{+} \mathrm{m} / \mathrm{z}$ : 424.1280, found: 424.1281 .

\section{N-(4-methoxyphenyl)-2-(4-(3-methyl- [1,2,4]triazolo[3,4-a]phthalazin-6-yl)phenyl) acetamide (8f)}

White solid, yield 66.2 \%; Mp: 178-180 ${ }^{\circ} \mathbf{C}$; Rf 0.40 (methanol: dichloro methane, $0.1: 1.0) ;{ }^{1} \mathrm{H}$ NMR (400 $\mathrm{MHz}, \mathrm{DMSO}) \delta 10.13(\mathrm{~s}, 1 \mathrm{H}), 8.55(\mathrm{~d}, J=8 \mathrm{~Hz}, 1 \mathrm{H})$, $8.04(\mathrm{~d}, J=4 \mathrm{~Hz}, 1 \mathrm{H}), 7.84(\mathrm{~s}, 1 \mathrm{H}), 7.68(\mathrm{~d}, \mathrm{~J}=8 \mathrm{~Hz}$, $2 \mathrm{H}), 7.59(\mathrm{~d}, J=4 \mathrm{~Hz}, 2 \mathrm{H}), 7.54(\mathrm{~d}, J=8 \mathrm{~Hz}, 2 \mathrm{H})$, $6.88(\mathrm{~d}, J=12 \mathrm{~Hz}, 2 \mathrm{H}), 3.77(\mathrm{~s}, 2 \mathrm{H}), 3.71(\mathrm{~s}, 3 \mathrm{H})$, $2.69(\mathrm{~s}, 3 \mathrm{H}) .{ }^{13} \mathrm{C}$ NMR (400 MHz, DMSO) $\delta 168.7$, 156.0, 155.2, 138.1, 133.8, 132.4, 132.3, 130.8, 129.4, 128.7, 123.3, 122.5, 122.3, 120.6, 113.8, 55.1, 43.0, 9.5. HR-MS(ESI): Calcd. $[\mathrm{M}+\mathrm{H}]^{+} \mathrm{m} / \mathrm{z}: 424.1280$, found: 424.1285 .

\section{N-(2-chlorophenyl)-2-(4-(3-methyl- [1,2,4]triazolo[3,4-a]phthalazin-6-yl)phenyl) acetamide $(8 \mathrm{~g})$}

Brown solid, yield 78.2\%; Mp: 167-169 ${ }^{\circ} \mathbf{C}$; $\mathrm{R}_{\mathrm{f}} 0.50$ (methanol: dichloro methane, $0.1: 1.0) ;{ }^{1} \mathrm{H}$ NMR (400 MHz, DMSO) $\delta 9.62(\mathrm{~s}, 1 \mathrm{H}), 8.37(\mathrm{~d}, \mathrm{~J}=8 \mathrm{~Hz}, 1 \mathrm{H})$, $7.87-7.83(\mathrm{~m}, 1 \mathrm{H}), 7.67-7.63(\mathrm{~m}, 2 \mathrm{H}), 7.54-7.48$ $(\mathrm{m}, 3 \mathrm{H}), 7.41(\mathrm{~d}, J=4 \mathrm{~Hz}, 2 \mathrm{H}), 7.32-7.29(\mathrm{~m}, 1 \mathrm{H})$, 7.15 - $7.11(\mathrm{~m}, 1 \mathrm{H}), 7.02-6.98(\mathrm{~m}, 1 \mathrm{H}), 3.70(\mathrm{~s}, 2 \mathrm{H})$, $2.30(\mathrm{~s}, 3 \mathrm{H}) .{ }^{13} \mathrm{C}$ NMR (100 MHz, DMSO) $\delta 170.1$, 155.2, 151.3, 146.3, 143.4, 139.4, 135.9, 135.1, 132.9, $132.8,130.3,130.1,127.2,126.9,124.9,123.2,121.6$, 121.4, 120.2, 115.3, 42.7, 9.1. HR-MS(ESI):Calcd. $[\mathrm{M}+\mathrm{H}]^{+} \mathrm{m} / \mathrm{z}$ : 428.0764, found: 428.0769.

\section{$N$-(3-chlorophenyl)-2-(4-(3-methyl-[1,2,4]- triazolo[3,4-a]phthalazin-6-yl)phenyl)acetamide (8h)}

Brown solid, yield 68.12\%; Mp: $165-167{ }^{\circ} \mathbf{C} ; \mathrm{R}_{\mathrm{f}} 0.50$ (methanol: dichloro methane, 0.1: 1.0); ${ }^{1} \mathrm{H}$ NMR (400 $\mathrm{MHz}, \mathrm{DMSO}) \delta 10.42$ (s, $1 \mathrm{H}), 8.56(\mathrm{~d}, J=8 \mathrm{~Hz}, 1 \mathrm{H})$, 8.06 - $8.02(\mathrm{~m}, 1 \mathrm{H}), 7.87$ - $7.79(\mathrm{~m}, 2 \mathrm{H}, \mathrm{ArH}), 7.68$ $7.63(\mathrm{~m}, 3 \mathrm{H}), 7.57(\mathrm{~d}, J=4 \mathrm{~Hz}, 2 \mathrm{H}), 7.46(\mathrm{t}, J=8 \mathrm{~Hz}$, $2 \mathrm{H}), 7.33(\mathrm{t}, J=8 \mathrm{~Hz}, 1 \mathrm{H}), 7.10(\mathrm{~d}, J=8 \mathrm{~Hz}, 1 \mathrm{H})$, 3.80 (s, 2H), 2.69 (s, 3H). ${ }^{13} \mathrm{C}$ NMR (100 MHz, DMSO) $\delta 169.9,155.2,152.2,146.0,143.6,139.7$, $135.9,135.5,132.7,132.6,130.2,130.1,128.2,126.9$, $124.9,122.9,121.6,121.4,121.2,115.9,42.9,9.3$. HR-MS(ESI): Calcd. [M+H] $]^{+} \mathrm{m} / \mathrm{z}$ : 428.0764, found: 428.0765 .

\section{N-(4-chlorophenyl)-2-(4-(3-methyl- [1,2,4]triazolo[3,4-a]phthalazin-6- yl)phenyl)acetamide (8i)}

Brown solid, yield $75.22 \%$; Mp: $169-171{ }^{\circ} \mathbf{C}$; $\mathrm{R}_{\mathrm{f}} 0.50$ (methanol: dichloro methane, 0.1: 1.0);

1H NMR (400 MHz, DMSO) $\delta 10.36$ (s, 1H), 8.56 $(\mathrm{d}, J=8 \mathrm{~Hz}, 1 \mathrm{H}), 8.04$ (p, $J=4 \mathrm{~Hz} 1 \mathrm{H}), 7.83(\mathrm{~d}, J=$ 
$4 \mathrm{~Hz}, 2 \mathrm{H}), 7.68-7.63(\mathrm{~m}, 4 \mathrm{H}), 7.57(\mathrm{~d}, J=8 \mathrm{~Hz}, 2 \mathrm{H})$, 7.35 (d, $J=8 \mathrm{~Hz}, 2 \mathrm{H}), 3.79$ (s, 2H), 2.68 (s, 3H).

${ }^{13} \mathrm{C}$ NMR (100 MHz, DMSO) $\delta 169.9,155.1,152.1$, 145.9, 143.1, 139.1, 135.6, 135.1, 132.7, 132.5, 130.1, $129.9,127.1,126.8,124.8,122.9,121.1,120.9,120.1$, 115.2, 42.9, 9.2.

HR-MS(ESI): Calcd. [M+H] ${ }^{+} \mathrm{m} / \mathrm{z}: 428.0764$, found: 428.0766 .

\section{Procedures for evaluation of anticancer and antimicrobial activities}

\section{MTT cell proliferation assay method}

The present compounds were tested on HCT116 cell lines using MTT cell proliferation assay method ${ }^{33}$ (Table 1). The HCT-116 cell line was obtained from National Centre for Cell Science (NCCS), Pune (India) and cultivated in Dulbecco's modified Eagle's red medium (DMEM) (Sigma Life Science, USA) containing $10 \%$ fetal bovine serum (FBS). The cells (2000 cells per well) were seeded in a 96-well microplate containing $100 \mu \mathrm{L}$ of DMEM $+10 \%$ FBS medium per well and incubated at $37{ }^{\circ} \mathrm{C}$ with $5 \% \mathrm{CO}_{2}$. The cells were treated with different concentrations of compounds up to $72 \mathrm{~h}$ for every 24 $\mathrm{h}$ interval. Controls were maintained with $0.5 \%$ DMSO. After $72 \mathrm{~h}$ treatment, $5 \mu \mathrm{L}$ of MTT (3-(4,5dimethylthiazol-2-yl)-2,5-diphenyltetrazolium bromide) reagent ( $\mathrm{R} \& \mathrm{D}$ Systems, USA) along with $45 \mu \mathrm{L}$ of phenol red-free DMEM (Sigma Life Science, USA) without FBS was added to each well and plates were incubated at $37{ }^{\circ} \mathrm{C}$ with $5 \% \mathrm{CO}_{2}$ for $4 \mathrm{~h}$. After that, $50 \mu \mathrm{L}$ of solubilisation buffer (R \& D Systems, USA) was added to each well to dissolve the coloured formazan crystals produced by the reduction of MTT. After $24 \mathrm{~h}$ the optical density was measured at $550 \mathrm{~nm}$ using a micro plate reader (Bio-Rad, USA).

\section{Antimicrobial activity}

The antimicrobial activity of the compounds was evaluated by agar well diffusion protocol ${ }^{40}$ against a panel of human pathogens (Table 2). Nutrient agar media was used for cultivating the test bacteria. Nutrient agar media was sterilized at $15 \mathrm{lbs}$ pressure at $121^{\circ} \mathrm{C}$ for 15 minutes, cooled and inoculated with test bacteria. After thoroughly mixing, the inoculated media was poured into Petri plates under aseptic condition and allow them to solidify, wells of about 6 $\mathrm{mm}$ diameter were made in each Petri plate with the help of sterilized cork borer. The compounds were dissolved in DMSO at a concentration of $1 \mathrm{mg} / 1 \mu \mathrm{L}$ about $200 \mu \mathrm{L}$ of dissolved compounds were added to each well. Adding DMSO alone to wells served a control. The commercially available tetracycline in DMSO serves as a positive control. The inoculated plates were incubated at $37^{\circ} \mathrm{C}$ temperature for $24 \mathrm{~h}$, the zone of inhibition produced by each compound was measured in centimetres $(\mathrm{Cm})$.

\section{Acknowledgements}

We are grateful to DST for providing FIST LevelI funding for the Department of Chemistry and thankful to Acharya Nagarjuna University, NNagar522510, Guntur, AP-India for constant support.

\section{Conflict of interest}

The authors declare no conflict of interest

\section{References}

1- R. Siegel, C. De Santis, A. Jemal, Colorectal cancer statistics - 2014, CA Cancer J., 2014, 64(2), 104.

2- World Cancer Report (2014).

3- D. E. Chavez, D. A. Parrish New heterocycles from tetrazines and oxadiazoles, J. Heterocycl. Chem., 2009, 46, 88-90.

4- G. Bold, K. H. Altmann, J. Frei, M. Lang, P. W. Manley, P. Traxler, B. Wietfeld, J. Bruggen, E. Buchdunger, R. Cozens, S. Ferrari, P. Furet, F. Hofmann, G. MartinyBaron, J. Mestan, J. Rosel, M. Sills, D. E. L. Stover, M. A. A. Hashash, F. Acemoglu, E. Boss, R. Emmenegger, L. Lasser, E. Masso, R. Roth, C. Schlachter, W. Vetterli, D. Wyss, J. Wood, M, New anilinophthalazines as potent and orally well-absorbed inhibitors of the VEGF receptor tyrosine kinases useful as antagonists of tumour-driven angiogenesis, J. Med. Chem., 2000, 43, 2310-2323.

5- G. Strappaghetti, C. Brodi, G. Giannaccini, L. Betti, New 4-(4-methyl-phenyl) phthalazine$1(2 H)$-one derivatives and their effects on $\alpha_{1}$-receptors, Bioorg. Med. Chem. Lett., 2006, 16, 2575-2579.

6- A. D. Lebsack, J. Gunzner, B. Wang, R. Pracitto, H. Schaffhauser, A. Santini, J. Aiyar, R. Bezverkov, B. Munoz, W. Liuc, S. Venkatraman, Identification and synthesis of $[1,2,4]$ triazolo[ $[3,4-a]$ phthalazinederivatives as high-affinity ligands to the $\alpha_{2} \delta-1$ subunit of voltage-gated calcium channel, Bioorg. Med. Chem. Lett., 2004, 14, 2463-2467.

7- T. Haack, R. Fattori, M. Napoletano, F. Pellacini, G. Fronza, G. Raffaini, F. Ganazzoli, Phthalazine PDE IV inhibitors: a conformational study of some 6-methoxy-1,4disubstituted derivatives, Bioorg. Med. Chem., 2005, 13, 4425-4433

8- E. L. Piatnitski, M. A. J. Duncton, A. S. Kiselyov, R. Katoch-Rouse, D. Sherman, D. L. Milligan, C. Balagtas, W. C. Wong, J.

Kawakami, J. F. Doody, Arylphthalazines: Identification of a new phthalazine chemotype as inhibitors of VEGFR kinase, Bioorg. Med. Chem. Lett., 2005, 15, 4696-4698.

9- K. A. Menear, C. Adcock, F. C. Alonso, K. Blackburn, L. Copsey, J. Drzewiecki, A. Fundo, A. Le Gall, S. Gomez, H. Javaid, C. F. Lence, N. M. B. Martin, C. Mydlowski, 
G. C. M. Smith, Novel alkoxybenzamide inhibitors of poly (ADP-ribose) polymerase, Bioorg. Med. Chem. Lett., 2008, 18, 3942-3945.

10- C. K. Ryu, E. R. Park, Mi-Young Ma, JiHeeNho, Synthesis and antifungal activity of 6-arylamino-phthalazine-5,8-diones and 6,7bis(arylthio)-phthalazine-5,8-diones, Bioorg. Med. Chem. Lett., 2007, 17, 2577-2580.

11- C. A. Gandolfi, G. Beggiolin, E. Menta, M. Palumbo, C. Sissi, S. Spinelli, F. Johnson, Chromophore-Modified Antitumor Anthracenediones: Synthesis, DNA Binding, and Cytotoxic Activity of 1,4-

Bis[(aminoalkyl)amino] benzo[g]phthalazine5,10-diones, J. Med. Chem., 1995, 38, 526-536.

12- E. M. Kassem, M. M. Kamel, M. El-zahar, New 1(2H)-phthalazinones of potential antimicrobial activity, Pharmazie, 1990, 45, 215-216.

13- St. Dima, M. Caprosu, M. Ungureanu, G. Grosu, M. Petrovanu, Nouveaux derives de la 1a 1-methyl-phthalazinium ylides doues d'activites antimicrobiennes et fongistatiques, Ann. Pharm. Fr., 1999, 57, 415-416.

14- S. Ito, K. Yamaguchi, Y. Komoda, Structural Confirmation of the Nitration Product of the 1(2H)-Phthalazinone as the 2-Nitro-1 $(2 \mathrm{H})$ phthalazinone, Chem. Pharm. Bull., 1992, 40, 3327-3329.

15- T. Haack, R. Fattori, M. Napoletano, F. Pellacini, G. Fronza, G. Raffaini, F. Ganazzoli, Phthalazine PDE IV inhibitors: Conformational study of some 6-methoxy-1,4-disubstituted derivatives, Bioorg. Med. Chem., 2005, 13, 4425-4433.

16- G Heinisch, and H. Frank, Pharmacologically Active Pyridazine Derivatives part 1: Progress in Medicinal Chemistry; ed. By G.P. Ellis; Elsevier: Amsterdam, The Netherlands 1990, 27, pp. 1-50.

17- G Heinisch, and H. Frank, Pharmacologically Active Pyridazine Derivatives part 2: Progress in Medicinal Chemistry; ed. By G.P. Ellis; Elsevier: Amsterdam, The Netherlands 1992, 29, pp. 141-184.

18- A. Melikian, G. Schiewer, J. P. Chambon, C. G. Wermuth, Condensation of muscimol or thiomuscimol with aminopyridazines yields GABA-A antagonists, J. Med. Chem., 1992, 35, 4092-4097.

19- M. Napoletano, G. Norcini, F. Pellacini, G. Morazzoni, P. Ferlenga, L. Pradella, Phthalazine PDE4 inhibitors. Part 2: The synthesis and biological evaluation of 6-methoxy-1,4-disubstituted derivatives, Bioorg. Med. Chem. Lett., 2001, 11, 33-37.

20- Jill. K. Robinson, Mark. J. Bollinger, and W. M. John, Luminol/H2O2 Chemiluminescence Detector for the Analysis of Nitric Oxide in Exhaled Breath, Anal. Chem., 1999, 22, 5131-5136.
21- H. Arakawa, J. IshidaJ, M. Yamaguchi, M. Nakamura, Chemiluminescent Products of Reaction between $\alpha$-Keto Acids and 4, 5Diaminophthalhydrazide, Chem. Pharm. Bull. , 1990, 38, 3491-3493.

22- H. Arakawa, J. IshidaJ, M.Yamaguchi, M. Nakamura, A New Chemiluminogenic Substrate for N-Acetyl- $\beta$-D-glucosaminidase, 4'-(6'-

Diethylaminobenzofuranyl)phthalylhydrazidoN-acetyl- $\beta$-D-glucosaminide, Chem. Pharm. Bull., 1991, 39: 411-416.

23- K. Samoto, Y. Ohkura, Ring expansion reaction of 1,3-dithiolanes and 1,3-oxathiolanes using tellurium tetrachloride, Chem. Lett., 1990, 19, 1323-1326.

24- R. T. Buckler, H. E. Hartzler, E. Kurchacova, G. Nichols, B. M. Phillips, Synthesis and antiinflammatory activity of some 1,2,3- and 1,2,4-triazolepropionic acids, J. Med. Chem., 1978, 21, 1254-1260.

25- R. Alvarez, S. Velazquez, A. S. Felix, S. Aquaro, E. D. Clercq, C. F. Perno, A. Karlsson, J. Balzarini, M. J. Camarasa, 1,2,3-Triazole-[2,5-Bis-O-(tertbutyldimethylsilyl)-Beta-D-ribofuranosyl]-3'spiro-5"-(4"-amino- 1",2"-oxathiole 2",2"dioxide) (TSAO) Analogs: Synthesis and AntiHIV-1 Activity, J. Med. Chem., 1994, 37, 4185-4195.

26- M. J. Genin, D. A. Allwine, D. J. Anderson, M. R. Barbachyn, D. Edward Emmert, S. A. Garmon, D. A. Graber, K. C. Grega, J. B. Hester, D. K. Hutchinson, J. Morris, R. J. Reischer, C. W. Ford, G. E. Zurenko, J. C. Hamel, R. D. Schaad, D. Stapert, B. H. Yagi, Substituent Effects on the Antibacterial Activity of Nitrogen-Carbon-Linked (Azolylphenyl)oxazolidinones with Expanded Activity Against the Fastidious Gram-Negative Organisms Haemophilus influenza and Moraxella catarrhalis, J. Med. Chem., 2000, 43, 953-970.

27- F. Reck, F. Zhou, M. Girardot, G. Kern, C. J. Eyermann, R. R. Ramsay, N. J. Hales, M. B. Gravestock, Identification of 4-Substituted 1,2,3-Triazoles as Novel Oxazolidinone Antibacterial Agents with Reduced Activity against Monoamine Oxidase A, J. Med. Chem., 2005, 48, 499-506.

28- A. C. Cunha, J. M. Figueiredo, J. L. M. Tributino, A. L.P. Miranda, H. C. Castro, R. B. Zingali, C. M. A. Fraga, M. C. B. V. de Souza, V. F. Ferreira, E. Barreiro, Antiplatelet properties of novel N-substituted-phenyl-1,2,3triazole-4-acyl hydrazone derivatives, J. Bioorg. Med. Chem., 2003, 11, 2051-2059.

29- S. A. Shahzad, Md. Yar, Z. A. Khan, L. Shahzadi, Sd. Ali. R. Naqvi, A. Mahmood, S. Ullah, A. J. Shaikh, T. A. Sherazi, A. T. Bale, J. Kukułowicz, M. Bajda, Identification of 1,2,4-triazoles as new thymidine phosphorylase 
inhibitors: Future anti-tumour drugs, bioorganic chemistry, 2019, 85, 209-220.

30- S. Akin, H. Ayaloglu, E. Gultekin, A. Colak, O. Bekircan, M. Y. Akatin, Synthesis of 1,2,4triazole-5-on derivatives and determination of carbonic anhydrase II isoenzyme inhibition effects, bioorganic chemistry, 2018, 83, 170-179.

31- J. Chen, Z. Ding, Y. Peng, F. Pan, Li. Jianjun, L. Zou, Y. Zhang, H. Liang, HIF-1 $\alpha$ Inhibition Reverses Multidrug Resistance in Colon Cancer Cells via Down regulation of MDR1/PGlycoprotein, Plos. One., 2014, 9, e98882.

32- K. Harris, X. Gao, S. Huerta-Yepez, P. Kapur, S. Huerta, HIF-1 $\alpha$ and Resistance to Ionizing Radiation in Rectal Cancer, Surgery Curr. Res., 2014, 4, 200.

33- N. Ramesh, M. Gangadhara Rao, V. Ravi, V. Umamaheswara Rao, B. Hari Babu, Mercuric chloride catalyzed synthesis of some anticancer 2-aryl-2,3-dihydroquinizolin-4(1H)ones, Med Chem Res., 2016, 25, 1945-1951.

34- N. Ramesh, M. Gangadhara Rao, M. Tirumala, V. Umamaheswara Rao, B. Hari Babu, Synthesis, Anticancer and Antioxidant Evaluation of Some New 2-Aryl and 2Pyrazole-2,3-dihydroquinazolin-4(1H)-ones, Asian J. Chem., 2016, 28, 1321-1324.

35- O. Surendranatha Reddy, Ch. Venkata Surya Narayana, K. J. P. Narayana, V. Anuradha, B. Hari Babu, Synthesis and cytotoxic evaluation for some new 2,5-disubstituted pyrimidine derivatives for anticancer activity, Med. Chem. Res., 2015, 24, 1777-1788.

36- O. Surendranatha Reddy, Ch. Venkata Surya Narayana, N. Sharmila, G. V. Ramana, V. Anuradha, B. Hari Babu, Synthesis and Cytotoxic Evaluation for Some New Dihydropyrimidinone Derivatives for Anticancer Activity, Lett. Drug Des. Discov., 2013, 10, 699-705.

37- Q. R. Zhang, D. Q. Xue, P. He, K. P. Shao, P. J. Chen, Y. F. Gu, J. L. Ren, L. H. Shan, H. M. Liu, Synthesis and antimicrobial activities of novel 1,2,4-triazolo [3,4-a] phthalazine derivatives, Bioorg. \& Med. Chem. Lett., 2014, 24, 1236-1238.

38- X. Y. Sun, C. Hu, X. Q. Deng, C. X. Wei, Z. G. Sun, Z. S. Quan, Synthesis and antiinflammatory activity evaluation of some novel 6-alkoxy(phenoxy)-[1,2,4] triazolo[3,4a]phthalazine-3-amine derivatives, Eur. J. Med. Chem., 2010, 45, 4807-4812.

39- D. Q. Xue, X. Y. Zhang, C. J. Wang, L. Y. Ma, N. Zhu, P. He, K. P. Shao, P. J. Chen, Y. F. Gu, X. S. Zhang, C. F. Wang, C. H. Ji, Q. R. Zhang, H. M. Liu, Synthesis and anticancer activities of novel 1,2,4-triazolo[3,4-a] phthalazine derivatives, Eur. J. Med. Chem., 2014, 85, 235- 244.

40- J G Cappucino, N. Sherman, Microbiology, a laboratory manual; ed. by K. Churchman; Pearson Education Inc; New Delhi, 2004, pp. 282. 